Maischesystem anzupassen ist. Was die Malz-(Handels-)analyse als solche anbelangt, so muss man' sich streng an die vereinbarte Methode halten, um vergleichbare Resultate zu erzielen.

Weiterhin erscheint auch die Frage einer Erwägung wert, inwieweit die Differenzen in den Extraktangaben bei verschiedenen Konzentrationen auf die Bestimmung der Stammwürze eines Bieres aus Alkohol- und wirklichem Extraktgehalt von Einfluss sind, ab z. B. die Unstimmigkeiten, die auf diesem Gebiete herrschen, ausschliesslich auf die Felıler der alten Balling formel oder auch ganz oder teilweise darauf zurückzuführen sind, dass die Bestimmung des Extraktes und der Stammwürze bei wesentlich verschiedenen Extraktkonzentrationen erfolgt.

\title{
H. Lüers.
}

Über eine kurze Extraktbestimmung in der Gerste berichtet L. Heintz ${ }^{1}$ ). Das Verfahren besitzt gegenüber den bisher gebräuchlichen den Vorteil, dass es sich in etwa 5 Stdn. durchführen lässt. Die Analyse gestaltet sich folgendermafen:

1. Zur Herstellung des Malzauszuges wird helles Malzschrot im Verhältnis $1: 5$ mit destilliertem Wasser von $45^{\circ} \mathrm{C}$ eingemaischt, 10 Minuten stehen gelassen, blank filtriert und das Filtrat abgekühlt.

2. Zur Analyse gelangt geputzte Gerste, oder yom Kaufmuster die erste und zweite Sorte des Sortiersiebes. $25 \mathrm{~g}$ Gerstenfeinmehl werden mit $50 \mathrm{ccm}$ Malzauszug gut eingemaischt und sodann noch weitere $25 \mathrm{ccm}$ zugegeben. Auf diese Weise wird lästige Klumpenbildung vermieden. Dann wird 1 Stde. im. Wasserbade bei $50-52^{\circ} \mathrm{C}$ gemaischt und rasch auf $70-72^{\circ} \mathrm{C}$ erhitzt. Bei dieser Temperatur hält man $1 / 2$ Stde. and gibt zum Ersatz des verdampfenden Wassers $1 / 4$ Stde. nach Erreichen der Temperatur von $70^{\circ} 20 \mathrm{ccm}$ Wasser von $70^{\circ} \mathrm{zu}$. Hierauf wird das Maischbad zum Kochen gebracht, 1/4 Stde. in kräftigem Kochen erhalten, auf $70^{\circ} \mathrm{C}$ abgekühlt, in den Becher nochmals $25 \mathrm{ccm}$ Malzauszug gegeben und ${ }_{1 / 4}^{1 /}$ Stde. bei $70^{\circ}$ verzuckert. Nach raschem Abkühlen auf Zimmertemperatur und Aufwägen auf $225 \mathrm{~g}$ wird im Filtrat pyknometrisch der Extrakt ermittelt. Die Berechnung der Ausbeute erfolgt anf die bisher übliche Weise. Der Malzauszug wird den gleichen Temperatursteigerungen, wie die Analysenprobe selbst ausgesetzt, und nach dem Aufwägen auf sein ursprüngliches Gewicbt und Filtration pyknometrisch auf Extrakt uutersucht.

H. L üers.

Bestimmung der Schwefelung im Malz. Zur qualitativen Prüfung werden nach 0. Rein ke ${ }^{2}$ ) $50 \mathrm{~g} \mathrm{Malz} \mathrm{(ganze} \mathrm{Körner)}+150 \mathrm{~cm}$ Wasser 3 Stdn. digeriert and $100 \mathrm{ccm}$ des Filtrates mit Zink und SchwefeIsäure behandelt. Nach 10-60 Minuten zeigt Bleipapier oft Bräunung an. Quantitativ bestimmt man die $\mathrm{SO}_{2}$, indem man $200 \mathrm{ccm}$ eines Malzauszuges $\left(100 \mathrm{~g}\right.$ Malzkörner $+300 \mathrm{ccm} \mathrm{H} \mathrm{H}_{2} \mathrm{O}$, nach $3 \mathrm{Stdn} .200 \mathrm{ccm}$ abfiltriert) mit Phosphorsäure im Kohlensäurestrom in vorgelegte Jod-

1) Wochenschrft. f. Branerei 33, 65 (1916). - 2) Chem. Ztg. 34, 1159 (1910). 